\title{
Genetics of tooth agenesis: how to move the field forward
}

\author{
Alexandre R. VIEIRA ${ }^{1}$ \\ 1- DDS, MSc, PhD, Department of Oral Biology, School of Dental Medicine, University of Pittsburgh, Pittisburgh, USA. \\ Corresponding address: Alexandre R. Vieira, 614 - Salk Hall - 3501 Terrace Street - Pittsburgh, PA, USA. e-mail: arv11@dental.pitt.edu
}

Tooth agenesis is the congenital lack of one or more of the deciduous or permanent teeth. Oligodontia is the agenesis of six or more permanent teeth (excluding third molars), whereas absence of less than six teeth is referred to as hypodontia. Anodontia refers to the absence of all deciduous and permanent teeth.

Tooth agenesis occurs more frequently among a few specific teeth (lateral incisors, second premolars, and third molars), with $10 \%$ to $25 \%$ of the population affected. Familial tooth agenesis is transmitted as an autosomaldominant, autosomal-recessive, or X-linked condition, but can also show no clear segregation pattern ${ }^{5}$. Affected members within a family often exhibit significant variability with regard to the location, symmetry, and number of teeth involved. Residual teeth can vary in size, shape, or rate of development. The permanent dentition is more affected than the primary dentition ${ }^{3}$.

In this issue of the Journal of Applied Oral Science, Wang, et al. ${ }^{8}$ (2013) report a study that combines a casecontrol analysis of a PAX9 variant and sporadic isolated tooth agenesis, and a case of anodontia. The case-control analysis probably suffers from low statistical power. The effects of PAX9 in sporadic cases of tooth agenesis are probably small and may be dependent of interaction with other genes, such as MSX $1^{7}$. Effects of $P A X 9$ have been detected when analysis included cases with preferential third molar agenesis ${ }^{1}$. One of the PAX9 variants studied, rs4904210, is a missense mutation (A240P) that is not considered to have any functional consequences since is highly prevalent in several populations (Figure 1).

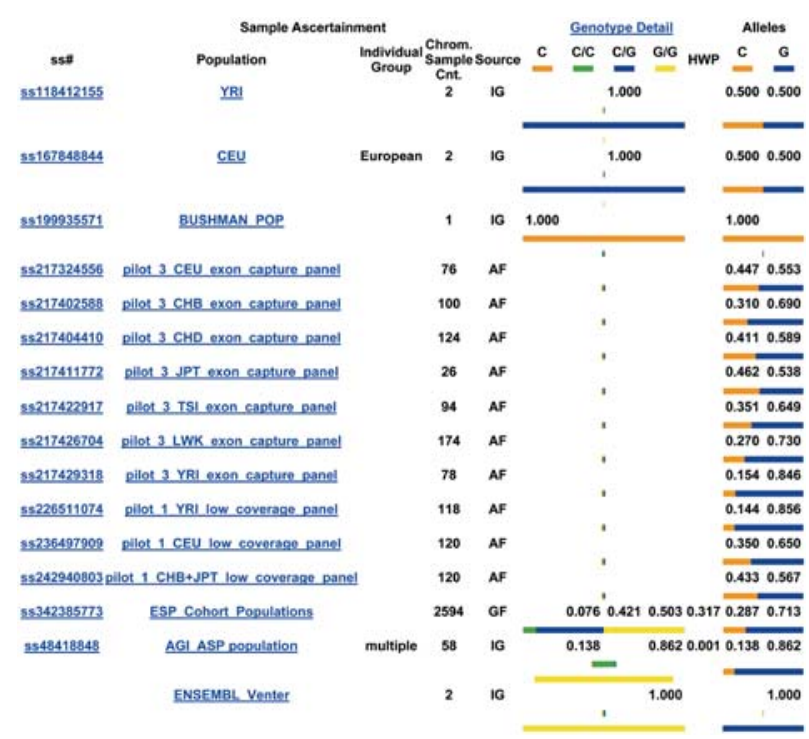

Source: NCBI - National Center for Biotechnology Information. dbSNP Short Genetic Variations. PAX9: rs4904210. Bethesda: NCBI; 2013 [cited 2013 June 20]. Available from:http://wwwncbi. nlm.nih.gov/projects/SNPsnp_ref cgi?rs=4904210

Figure 1- Allele and genotype frequencies of the PAX9 A240P mutation
The case of anodontia however rings more excitement to this report. The authors failed to find etiological mutations in four genes: PAX9, MSX1, AXIN2, and EDA. Sequencing candidate genes is not an unjustified first step, although no reports exist linking these genes with isolated anodontia, even when the whole PAX9 gene is deleted from one of the chromosomes ${ }^{6}$. The first three genes when mutated cause autosomal dominant forms of oligodontia (with at least one known MSX1 recessive form $)^{6}$. EDA has been linked to isolated $X$-linked recessive oligodontia, and whereas mutations in this gene cause ectodermal dysplasia, which can lead to anodontia or very severe oligodontia, sequencing EDA in a female case is not fully justified, unless the underlying hypothesis includes a chance of skewed $X$ chromosome inactivation.

A very appealing approach to be used for identifying the causal mutation of the anodontia case presented by Wang, et al. ${ }^{8}$ (2013) would be whole exome sequencing ${ }^{2}$. This approach has been used in other craniofacial conditions, such as craniosynostosis ${ }^{4}$. Using this approach, the authors will likely unveil the mutation causing the sporadic anodontia in the case by testing only one or two DNA samples of good quality for a current cost of less than US $\$ 1,000$.

\section{References}

1- Bianch FJ, Oliveira TF, Saito CB, Peres RC, Line SR. Association between polymorphism in the promoter region (G/C-915) of PAX9 gene and third molar agenesis. J Appl Oral Sci. 2007;15:382-6. 2- Choi, Scholl UI, Ji W, Liu T, Tikhonova IR, Zumbo P, et al. Genetic diagnosis by whole exome capture and massively parallel DNA sequencing. Proc Natl Acad Sci U.S.A. 2009;106:19096-101. 3- D'Souza RN, Kapadia H, Vieira AR. Teeth. In: Stevenson RE, Hall JG. Human malformations and related anomalies. New York: Oxford University Press; 2009. p.425-65.

4- Sharma VP, Fenwick AL, Brockop MS, McGowan SJ, Goos JA, Hoogeboom AJ, et al. Mutations in TCF12, encoding a basic helixloop-helix partner of TWIST1, are a frequent cause of coronal craniosynostosis. Nat Genet. 2013;45:304-7.

5- Vieira AR. Oral clefts and syndromic forms of tooth agenesis as models for genetics of isolated tooth agenesis. J Dent Res. 2003;82:162-5.

6- Vieira AR. Genetics of congenital tooth agenesis. eLS. Chichester: John Wiley \& Sons; 2012. doi: 10.1002/9780470015902. a0023576.

7- Vieira AR, Meira R, Modesto A, Murray JC. MSX1, PAX9, and TGFA contribute to tooth agenesis in humans. J Dent Res. 2004;83:723-7.

8- Wang J, Xu Y, Chen J, Wang F, Huang R, Wu S, et al. PAX9 polymorphism and susceptibility to sporadic non-syndromic severe anodontia: a case-control study in southwest China. J Appl Oral Sci. $2013 ; 21: 256-64$. 